\title{
Editorial overview: Immunomodulation: Exploiting the circle between emotions and immunity: impact on pharmacological treatments
}

\section{Fulvio D'Acquisto}

Current Opinion in Pharmacology 2016, 29:viii-xii

For a complete overview see the Issue

Available online 26th July 2016

http://dx.doi.org/10.1016/j.coph.2016.07.008

1471-4892/Crown Copyright (C) 2016 Published by

Elsevier Ltd. All rights reserved.

\section{Fulvio D'Acquisto}

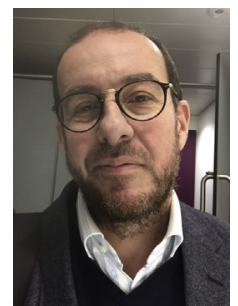

William Harvey Research Institute, Queen Mary University of London, Barts and the London School of Medicine and Dentistry, Queen Mary University of London, London, UK

e-mail: F.Dacquisto@qmul.ac.uk

Fulvio D'Acquisto is a professor of Immunopharmacology at the William Harvey Research Institute, Queen Mary University of London in UK. His current field of research (called Affective Immunology; http://www. affectiveimmunology.com) investigates the cross-talk between emotions and the immune system as a new venue for the treatment of autoimmune and inflammatory disorders.
Society is rapidly changing and so are many aspects of our daily living. From the ways we communicate with each other to the goods we consume, the most striking change we have witnessed in the last decades is the falling of barriers between apparently diverse parts of society and the merging of different cultures and points of view. We have called this phenomenon 'globalization' and, according to its definition, this is 'the process by which the world is becoming increasingly interconnected as a result of massively increased trade and cultural exchange'. How does globalization manifest in science and scientific research? The short answer is 'system biology' for example the understanding and analysis of biological phenomena through the lenses of different and yet complementary disciplines (mathematics combined with enzymology for instance) [1].

Translating these concepts into the world of pharmacology and drug discovery, one could say diseases are not just a straight line - one organ/ one cell/one signalling pathway - but rather a spider diagram where different 'factors and problems' all converge to a single point. With this in mind, it would be tempting to say that the benefits of combining different approaches to achieve an improved and safer pharmacological therapy should be a no brainer. In this section of Immunomodulation, we have been inspired by this concept and have explored the link between mind and body in general - the interconnectedness between emotional states and the immune system specifically — as a possible venue for an improved treatment of both immune and emotional disorders. I have named this area of research Affective Immunology (http://www.affectiveimmunology.com).

Why would the link between emotions and immunity be important for drug design? And why should pharmacologists, in particular, be investigating this area of research? The answers to these questions are multiple. First, some immunomodulatory therapies present serious emotional side effects (increased suicide idealization) [2,3], and others might provide a better therapeutic effect when administered with 'emotional modulators' (see later) [4]. Second, pharmacological therapies for mood disorders have been shown to be more effective when co-administered with immunomodulatory drugs in specific cohorts of patients [5-7].

What are the cellular and molecular mechanisms underlying the connection between emotions and immunity? We know first of all that the immune system plays a key role in maintaining the emotional system under check 
[8-10]. Studies in both experimental animals and humans have shown that a dysfunctional or absent immune system causes some emotional disorders including anxiety, depression, obsessive-compulsive disorders and increased risk of suicide [11-13]. In mice, the absence of immune cells causes significant changes in memory and cognition [14-16] and increases the animal's constitutive basal level of anxiety-like behavior $[17,18]$. These effects are linked to some mechanisms including the lack of homeostatic 'patrolling' exerted by the immune cells into the CNS through newly discovered lymphatic vessels $[19,20]$. Studies also suggest that the absence of $\mathrm{T}$ cells causes specific changes in the gene expression profile of the whole brain, and these have been associated with both anxiety disorders and neurodegenerative diseases [17]. Similar findings have been reported in humans as patients with either a deficient immune system (HIV) [21,22] or those with a hyper activated one (autoimmune diseases) are known to present a high incidence of emotional disorders [23-26].

Despite this evidence, the scientific community seems to be reluctant to appreciate the therapeutic value of these observations. As Lasselin and colleagues stated in their article, most people are still surprised to hear that 'immunity is tuned by one's emotions, personality and social status'. Therefore, it might come not as a surprise to know that a defective immune response could be treated by combined targeting of the emotional and immunological systems. As the authors suggested, this 'resistance' might be because emotional wellbeing is not well-defined and hence difficult to quantify. The absence of 'universal' and well-tested animal models for the study of emotional wellbeing might be another cause. Besides these considerations, I think that the resistance also lays on the fact that wellbeing is not just about how we feel but also about how we are, as suggested by the authors and others [27]. This is rather important as it suggests that therapies tackling 'how one is' or in other terms one's life style might be potentially useful when combined with standard immunomodulatory therapies.

Along these lines, a large body of evidence has shown a direct correlation between life style, oxidative stress and immune and inflammatory chronic diseases [28-30]. The article by Samina Salin discusses the role of oxidative stress in neuroinflammation and the fact that many psychiatric illnesses are reported to exhibit low plasma concentrations of a variety of antioxidants including glutathione, vitamin $E$ and coenzyme Q10. The idea that the combined administration of immunomodulators and changes in life-style might be beneficial for the therapy of immune and emotional disorders is indeed fascinating as Chiurchiu and Maccarone suggested. In their article, the authors focused their attention on bioactive lipids [31-33] and their dual role in immunity and emotions. From classical lipids like eicosanoids and phospholipids to more recent new entries such as specialized pro-resolving lipid mediators, the range of mediators that could be pharmacologically exploited for their dual pharmacological effects on emotional state $[34,35]$ and immunity [36,37] seems to be growing by the year. Of a particular note for the topic of this volume, the endocannabinoid anandamide is the prototype of lipid mediator that has remarkable effects on the immune system [38] and is equally effective in emotional disorders $[39,40]$ as its name (which means 'inner bliss') seems to suggest.

The idea of drugs with a potential double role in the immune and emotional systems is not entirely new. Neigh and Ali have provided an interesting overview of immunomodulatory drugs - from the classical steroid to the modern biologic against inflammatory cytokines that are proven for the treatment of post-traumatic stress disorders (PTSD). The authors also highlighted that several drugs used for the treatment of PTSD, including selective serotonin re-uptake inhibitors (SSRI), have been shown to exert anti-inflammatory effects on T-lymphocytes, dendritic cells, and neutrophils.

This simultaneous effect on both the immune and emotional system can also be achieved by co-therapy. In their article Rosenblat and co-authors describe the efficacy of co-therapy in the treatment of patients suffering from bipolar disorders. Similar to what has been found for PTSD or depression, a wide range of drugs including $N$ acetylcysteine, infliximab, pioglitazone, celecoxib, aspirin, and omega-3 polyunsaturated fatty acids have shown an antidepressant effect in bipolar disorders when administered adjunctively to conventional treatments.

Co-therapies have also been particularly useful in the context of immunomodulatory treatment. Indeed, Kovacs and co-authors provided an extensive overview of the side effects on the emotional system of a powerful antiviral and anticancer drug: interferon-alpha [41]. As stated by the authors, a staggering $10 \%-40 \%$ of patients receiving this therapy develop a full depressive disorder syndrome that can include suicidal ideation, aboulia, lack of motivation, social withdrawal, guilt, anhedonia, irritability, anxiety, and crying $[42,43]$. Luckily, the side effects of this drug seem to be tapered by the co-treatment with traditional drugs such as SSRI [44] or novel ones such as a diet rich in omega-3 polyunsaturated fatty acids [45].

Is there any other approach to co-therapies? As we have just started to better appreciate the colloquium between the brain and immune cells, an extra level of control has been taking the center stage in the scientific arena: the gut-brain axis or - to be more precise - the gut-brainimmune system triangle. The colloquium has now been transformed into colloquia. The discoveries emerging from this field of research are bewildering, to say the least [46-48]. As Hayley and colleagues pointed out, bacterial communities generally present in the gut are 
Figure 1

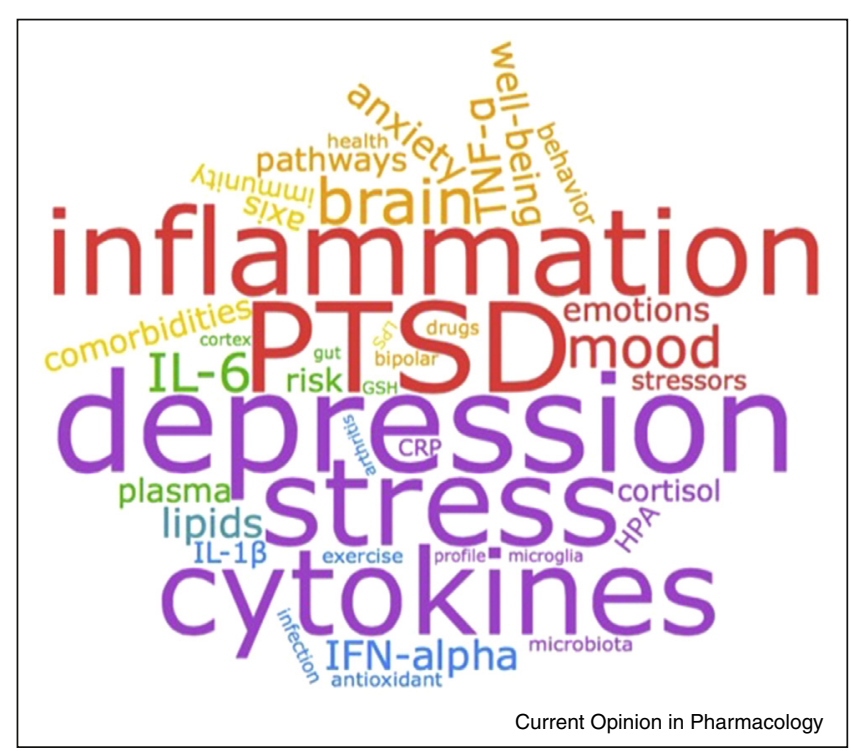

Affective Immunology: where immunity and emotions converge. Tag-cloud analysis of the most common terms used in the contributions made to this volume of Current Opinion in Pharmacology dedicated to Immunomodulation. Terms like "depression", "PTSD", "inflammation", "stress" and "cytokines" had the highest score (cited more than 70 times) and might represent the common feature among all the topics that have been explored. Terms like "microglia", "GSH", and "gut" were the least used (cited 10 times) and might represent themes that are developing or are more relevant to specific aspects.

sensitive to a variety of challenges, including stressors the stressors being of emotional nature (the most famous 'gut feelings') or a pathogenic factor for the immune system. In their view, the gut is the real link between the brain and the immune system as evidenced by the fact that the benefits of microbiota-targeting compounds on mood states could be related to their actions on immune processes. Prebiotics and pro-biotics are the new classes of drugs currently under investigation for their use in immune and psychiatric disorders [49-51].

Where do we move from here? Figure 1 shows a tag cloud analysis of the most common terms present in all the contributions to this volume and highlight the 'challenges' ahead of us. From my personal perspective, there are still two most important aspects that need to be explored. First, the role of emotions and immune cells as guardian of wellbeing. As Ruslan Medzhitov has highlighted in his recent reviews [52,53], the state of homoeostasis of the immune system cannot be just interpreted as absence of threats from the external environment. Emotions like stress or simply having a laugh are part of daily living, and as Steve Cole and colleagues has very elegantly shown, they directly steer the immune system towards specific tasks such as antibacterial defense pathway or antiviral response depending on social factors such as isolation [54-56]. Unraveling the molecular mechanisms regulating these events might provide an immense resource for pharmacologists to identify new molecules and drugs that can help maintain the host in a state of immunological equilibrium and possibly a healthy emotional state.

The other aspect that has not been thoroughly investigated is the contribution of emotional or immunological systems in controlling the transition between acute and chronic diseases. Indeed, it could be that the disturbance in emotional wellbeing is one of the leading causes that make acute inflammatory response transform into chronic autoimmune disorders. Similarly, change in the immune system might constitute the tipping point for a weak emotional state to become a chronic and morbid mood condition. The validation of this hypothesis would lead to an entirely new therapeutic protocol where co-therapy will be substituted by sequential therapies. Thus, a patient with high risk of mental disorders will be given treatment for the mental disorder first and an immunomodulatory therapy as a preventative of future flares. Similarly, patients with autoimmune diseases will be treated with immunosuppressants during the acute phase of the disease and then kept under 'emotional modulators' as soon as remission comes up.

Clearly, the validity of such protocols will require several clinical trials before a definite answer can be reached. In the meantime, I hope that this volume on immunomodulation will convince my pharmacologist colleagues as well as scientists from other disciplines to 'open their mind' and wonder if the disease they are studying could also be looked at from the perspective of an apparently distant area of research. As history and current events have convincingly shown 'separation' is never the best and most convenient choice to address a problem. In life as in science!

\section{Conflict of interest statement}

Nothing .declared.

\section{Acknowledgements}

I am very grateful to Dr Dianne Cooper and Dr Lucy Norling for their feedback and suggestions.

\section{References}

1. Kirschner MW: The meaning of systems biology. Cell 2005, 121:503-504.

2. Bosche $\mathrm{K}$, Weissenborn $\mathrm{K}$, Christians $\mathrm{U}$, Witzke $\mathrm{O}$, Engler $\mathrm{H}$, Schedlowski M, Hadamitzky M: Neurobehavioral consequences of small molecule-drug immunosuppression. Neuropharmacology 2015, 96:83-93.

3. Fireman M, DiMartini AF, Armstrong SC, Cozza KL: Immunosuppressants. Psychosomatics 2004, 45:354-360.

4. Sarkar S, Schaefer M: Antidepressant pretreatment for the prevention of interferon alfa-associated depression: a systematic review and meta-analysis. Psychosomatics 2014, 55:221-234. 
5. Andrade C: Antidepressant augmentation with antiinflammatory agents. J Clin Psychiatry 2014, 75:975-977.

6. Ghanizadeh A, Hedayati A: Augmentation of citalopram with aspirin for treating major depressive disorder, a double blind randomized placebo controlled clinical trial. Antiinflamm Antiallergy Agents Med Chem 2014, 13:108-111.

7. $\mathrm{Na} \mathrm{KS}$, Lee KJ, Lee JS, Cho YS, Jung HY: Efficacy of adjunctive celecoxib treatment for patients with major depressive disorder: a meta-analysis. Prog Neuropsychopharmacol Biol Psychiatry 2014, 48:79-85.

8. Barak $\mathrm{Y}$ : The immune system and happiness. Autoimmun Rev 2006, 5:523-527.

9. D'Acquisto F, Rattazzi L, Piras G: Smile - it's in your blood! Biochem Pharmacol 2014, 91:287-292.

10. Brod S, Rattazzi L, Piras G, D'Acquisto F: 'As above, so below' examining the interplay between emotion and the immune system. Immunology 2014, 143:311-318.

11. Rook GA, Lowry CA, Raison CL: Lymphocytes in neuroprotection, cognition and emotion: is intolerance really the answer? Brain Behav Immun 2011, 25:591-601.

12. McCray CJ, Agarwal SK: Stress and autoimmunity. Immunol Allergy Clin North Am 2011, 31:1-18.

13. Stojanovich L: Stress and autoimmunity. Autoimmun Rev 2010, 9:A271-A276.

14. Kipnis J, Gadani S, Derecki NC: Pro-cognitive properties of T cells. Nat Rev Immunol 2012, 12:663-669.

15. Walsh JT, Kipnis J: Regulatory T cells in CNS injury: the simple, the complex and the confused. Trends Mol Med 2011, 17:541-547.

16. Schwartz M, Kipnis J: A conceptual revolution in the relationships between the brain and immunity. Brain Behav Immun 2011, 25:817-819.

17. Rattazzi L, Piras G, Ono M, Deacon R, Pariante CM, D'Acquisto F: CD4(+) but not CD8(+) T cells revert the impaired emotional behavior of immunocompromised RAG-1-deficient mice. Trans/ Psychiatry 2013, 3:e280.

18. Rattazzi L, Cariboni A, Poojara R, Shoenfeld $Y$, D'Acquisto F: Impaired sense of smell and altered olfactory system in RAG1(-) immunodeficient mice. Front Neurosci 2015, 9:318.

19. Louveau A, Harris TH, Kipnis J: Revisiting the mechanisms of CNS immune privilege. Trends Immunol 2015, 36:569-577.

20. Louveau A, Smirnov I, Keyes TJ, Eccles JD, Rouhani SJ, Peske JD, Derecki NC, Castle D, Mandell JW, Lee KS et al.: Structural and functional features of central nervous system lymphatic vessels. Nature 2015, 523:337-341.

21. Gallego L, Barreiro P, Lopez-lbor JJ: Psychopharmacological treatments in HIV patients under antiretroviral therapy. AIDS Rev 2012, 14:101-111.

22. Jacobs N: HIV and mental health. Ment Health Today 2011:28-32.

23. Leyhe $T$, Mussig $\mathrm{K}$ : Cognitive and affective dysfunctions in autoimmune thyroiditis. Brain Behav Immun 2014, 41:261-266.

24. Isaac ML, Larson EB: Medical conditions with neuropsychiatric manifestations. Med Clin North Am 2014, 98:1193-1208.

25. Bacconnier L, Rincheval N, Flipo RM, Goupille P, Daures JP, Boulenger JP, Combe B: Psychological distress over time in early rheumatoid arthritis: results from a longitudinal study in an early arthritis cohort. Rheumatology (Oxford) 2015 54:520-527.

26. Denton FJ, Sharpe L, Schrieber L: Cognitive bias in systemic lupus erythematosus. Eur J Pain 2005, 9:5-14.

27. Steptoe A, Deaton A, Stone AA: Subjective wellbeing, health, and ageing. Lancet 2015, 385:640-648.

28. Rani V, Deep G, Singh RK, Palle K, Yadav UC: Oxidative stress and metabolic disorders: pathogenesis and therapeutic strategies. Life Sci 2016, 148:183-193.
29. Bondia-Pons I, Ryan L, Martinez JA: Oxidative stress and inflammation interactions in human obesity. J Physiol Biochem 2012, 68:701-711.

30. Nafar M, Sahraei Z, Salamzadeh J, Samavat S, Vaziri ND: Oxidative stress in kidney transplantation: causes, consequences, and potential treatment. Iran J Kidney Dis 2011, 5:357-372.

31. Nadjar A, Leyrolle Q, Joffre C, Laye S: Bioactive lipids as new class of microglial modulators: when nutrition meets neuroimunology. Prog Neuropsychopharmacol Biol Psychiatry 2016.

32. Tintut $Y$, Demer LL: Effects of bioactive lipids and lipoproteins on bone. Trends Endocrinol Metab 2014, 25:53-59.

33. Nagao K, Yanagita T: Bioactive lipids in metabolic syndrome. Prog Lipid Res 2008, 47:127-146.

34. Berger GE, Smesny S, Amminger GP: Bioactive lipids in schizophrenia. Int Rev Psychiatry 2006, 18:85-98.

35. Condray R, Yao JK: Cognition, dopamine and bioactive lipids in schizophrenia. Front Biosci (Schol Ed) 2011, 3:298-330.

36. Cabral GA, Ferreira GA, Jamerson MJ: Endocannabinoids and the immune system in health and disease. Handb Exp Pharmacol 2015, 231:185-211.

37. Dennis EA, Norris PC: Eicosanoid storm in infection and inflammation. Nat Rev Immunol 2015, 15:511-523.

38. Maccarrone M, Bab I, Biro T, Cabral GA, Dey SK, Di Marzo V, Konje JC, Kunos G, Mechoulam R, Pacher P et al:: Endocannabinoid signaling at the periphery: 50 years after THC. Trends Pharmacol Sci 2015, 36:277-296.

39. Ashton $\mathrm{CH}$, Moore PB: Endocannabinoid system dysfunction in mood and related disorders. Acta Psychiatr Scand 2011 124:250-261.

40. Moreira FA, Wotjak CT: Cannabinoids and anxiety. Curr Top Behav Neurosci 2010, 2:429-450.

41. Arico E, Belardelli F: Interferon-alpha as antiviral and antitumor vaccine adjuvants: mechanisms of action and response signature. J Interferon Cytokine Res 2012, 32:235-247.

42. Raison CL, Demetrashvili M, Capuron L, Miller AH: Neuropsychiatric adverse effects of interferon-alpha: recognition and management. CNS Drugs 2005, 19:105-123.

43. Schaefer M, Engelbrecht MA, Gut O, Fiebich BL, Bauer J Schmidt F, Grunze H, Lieb K: Interferon alpha (IFNalpha) and psychiatric syndromes: a review. Prog Neuropsychopharmacol Biol Psychiatry 2002, 26:731-746.

44. Ehret M, Sobieraj DM: Prevention of interferon-alphaassociated depression with antidepressant medications in patients with hepatitis $\mathbf{C}$ virus: a systematic review and metaanalysis. Int J Clin Pract 2014, 68:255-261.

45. Su KP, Matsuoka Y, Pae CU: Omega-3 polyunsaturated fatty acids in prevention of mood and anxiety disorders. Clin Psychopharmacol Neurosci 2015, 13:129-137.

46. Petra Al, Panagiotidou S, Hatziagelaki E, Stewart JM, Conti P, Theoharides TC: Gut-microbiota-brain axis and its effect on neuropsychiatric disorders with suspected immune dysregulation. Clin Ther 2015, 37:984-995.

47. El Aidy S, Dinan TG, Cryan JF: Immune modulation of the braingut-microbe axis. Front Microbiol 2014, 5:146.

48. Rogers GB, Keating DJ, Young RL, Wong ML, Licinio J, Wesselingh S: From gut dysbiosis to altered brain function and mental illness: mechanisms and pathways. Mol Psychiatry 2016, 21:738-748.

49. Liu X, Cao S, Zhang X: Modulation of gut-microbiota-brain axis by probiotics prebiotics, and diet. J Agric Food Chem 2015, 63:7885-7895.

50. Patterson E, Cryan JF, Fitzgerald GF, Ross RP, Dinan TG, Stanton C: Gut microbiota, the pharmabiotics they produce and host health. Proc Nutr Soc 2014, 73:477-489. 
51. Saulnier DM, Ringel $Y$, Heyman MB, Foster JA, Bercik $P$, Shulman RJ, Versalovic J, Verdu EF, Dinan TG, Hecht G et al.: The intestinal microbiome, probiotics and prebiotics in neurogastroenterology. Gut Microbes 2013, 4:17-27.

52. Kotas ME, Medzhitov R: Homeostasis, inflammation, and disease susceptibility. Cell 2015, 160:816-827.

53. Chovatiya R, Medzhitov R: Stress, inflammation, and defense of homeostasis. Mol Cell 2014, 54:281-288.
54. Fredrickson BL, Grewen KM, Algoe SB, Firestine AM, Arevalo JM, Ma J, Cole SW: Psychological well-being and the human conserved transcriptional response to adversity. PLOS ONE 2015, 10:e0121839.

55. Cole SW: Human social genomics. PLoS Genet 2014, 10:e1004601.

56. Cole SW: Social regulation of human gene expression: mechanisms and implications for public health. Am J Public Health 2013, 103(Suppl. 1):S84-S92. 